\title{
Ethics and SARS: lessons from Toronto
}

\author{
Peter A Singer, Solomon R Benatar, Mark Bernstein, Abdallah S Daar, Bernard M Dickens, \\ Susan K MacRae, Ross E G Upshur, Linda Wright, Randi Zlotnik Shaul
}

The SARS epidemic showed how easy it is for infectious diseases to spread round the world. Ethical as well as clinical issues need to be resolved to improve the response to the next epidemic

The outbreak of severe acute respiratory syndrome (SARS) in the Toronto area earlier this year forced medical and government workers to make hard choices, often with limited information and short deadlines. Healthcare providers were on the firing line, and were the people most affected by the disease. ${ }^{1}$ Decision makers had to balance individual freedoms against the common good, fear for personal safety against the duty to treat sick people, and economic losses against the need to contain the spread of a deadly disease. Such decisions have to be guided by both scientific knowledge and ethical considerations. The SARS outbreak showed that Canadian society was not fully prepared to deal with the ethical issues.

\section{Evaluating ethical issues}

We formed a working group to identify the key ethical issues and values most important for an analysis of ethical dimensions of the SARS epidemic. The final list of issues and values was agreed by a consensus process and found to have face validity and credibility. We then developed a framework for looking at the ethical implications of the SARS outbreak, identifying 10 key ethical values relevant to SARS (box), and five major ethical issues faced by decision makers.

We examined the underlying ethical values for the five major issues and drew lessons from how each was tackled. The following case studies illustrate the issues and are an amalgam of our experiences.

\section{Ethics of quarantine}

A medical clerk is asked by public health officials to remain at home in quarantine for 10 days because of possible exposure to SARS. She wants to comply but fears this could cost both her job and her apartment.

Five ethical values were associated with this issue, starting with individual liberty. Even the most highly valued individual liberties have to be balanced against

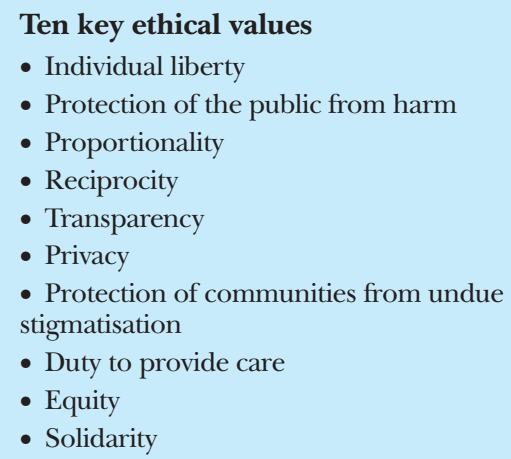

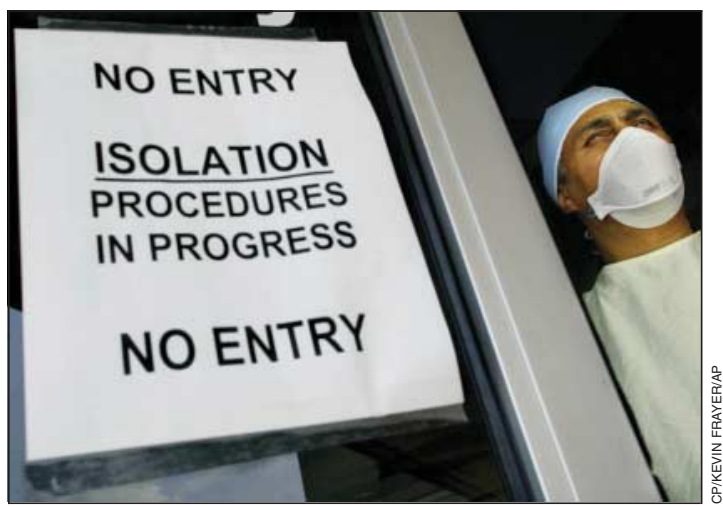

a second value-that of protecting the public from harm caused by the uncontrolled movements of people who may be infectious. ${ }^{2}$ Under the value of proportionality, authorities exercising public health powers should do so in a way that is relevant, legitimate, and necessary. They should use the least restrictive methods that are reasonably available to limit individual liberties and should apply restrictions without discrimination.

The value of transparency requires stakeholders to be fully informed about issues, including the risks and benefits, particularly if they affect their health, wellbeing, and personal liberty. Finally, the value of reciprocity requires society to ensure that those quarantined receive adequate care and do not suffer unfair economic penalties. ${ }^{3}$

\section{Privacy of personal information and public need to know}

A nurse at a hospital affected by SARS feels unwell and has a fever. After weighing the risk of having the disease against losing income and placing a burden of extra work on her colleagues, the nurse takes a commuter train to work. She is later found to have SARS. Medical officials choose not to name the nurse but use the media to warn people who may have been on her train that they should be tested for SARS.

Although the individual has a right to privacy, the state can over-ride this right if it would greatly help protect the public from serious harm. As a general rule, the privacy and confidentiality of individuals should be protected unless a well defined public health goal can be achieved by making personal information public.

In the initial stages of the outbreak, authorities named the woman who carried SARS to Canada from China, and her son, with the family's consent, because they believed it would provide additional public health benefit. Although public health officials took great pains to avoid linking ethnicity and illness, the linking of SARS with someone who had travelled from China,
University of

Toronto Joint Centre for

Bioethics,

88 College St,

Toronto, Canada

M5G 1L4

Peter A Singer Sun Life financial chair and director Abdallah S Daar director, programme in applied ethics and biotechnology

Susan K MacRae

deputy director

Linda Wright

bioethicist

Randi Zlotnik Shaul bioethicist

Department of Public Health Sciences, University of Toronto

Solomon R Benatar visiting professor

Division of

Neurosurgery,

University of

Toronto

Mark Bernstein

professor

Faculty of Law,

University of

Toronto

Bernard M Dickens Dr William M Scholl professor of health law and policy

Sunnybrook and Women's College Health Sciences Centre, Toronto Ross E G Upshur director, primary care research unit

Correspondence to: P A Singer peter.singer@ utoronto.ca

BMJ 2003;327:1342-44

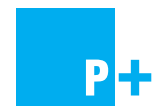

The decision making tool is available on bmi.com 
combined with the public's limited understanding of transmission, resulted in many people unnecessarily avoiding Chinese businesses.

Proportionality requires that private information be released only if there are no less intrusive means to protect the public health. For example, naming an individual or releasing a photograph could be justified if that person violates a quarantine order. Transparency can be achieved without naming individuals or communities. Indeed, the need to protect communities from undue stigma is an important lesson for future epidemics.

\section{Duty of care}

An intensive care nurse is afraid of contracting SARS at work and infecting her husband and three small children. She feels torn between the potential danger posed to her family and her professional duties.

For the first time in more than a generation, Canadian healthcare workers faced a deadly infectious disease for which there was no known effective treatment. Dozens of healthcare workers, many of them nurses, were infected with SARS because of their work. Some infected their families, and two died. Many others had to wear cumbersome equipment to protect themselves, causing appreciable discomfort, reducing their ability to work, and reducing contact with their patients.

Healthcare workers were forced to weigh serious and imminent health risks to themselves and their families against their duty to care for the sick. This duty is mainly determined by professional ethics. By analogy, firefighters do not have the freedom to choose whether to face a particularly bad fire and police officers do not get to select which dark alleys they walk down.

Nonetheless, these professional duties do have limits. For example, healthcare workers also have a professional obligation to maintain their own health and ability to provide care for patients. ${ }^{4}$ However, the nature of such limits is contested. Some believe that under dire circumstances professionals should have minimal self regard and pursue their duties at potential cost to their own lives. Others claim that it is unreasonable to demand extreme heroism as the norm, and even more unreasonable to demand that the lives of children and families should be held hostage to professional duties. How these two positions are balanced is intensely personal but has vital implications for handling pandemics.

The value of reciprocity requires healthcare institutions to support and protect healthcare workers, to help them cope with very stressful situations, to acknowledge their work in dangerous and difficult conditions, and to have workable plans for emergency situations. Ontario's minister of health acknowledged the heroic work of healthcare workers, and this was an important act of reciprocity. In addition, measures such as an insurance fund to cover healthcare workers who become sick or die through work should be considered for future epidemics. Although many doctors and nurses have supplemental insurance, other healthcare workers, such as technicians and clerks, do not.

We could not reach consensus on the issue of duty to care, particularly regarding the extent to which healthcare workers are obligated to risk their lives in delivering clinical care. This issue requires urgent attention from researchers, regulatory bodies, and the public.

\section{Collateral damage}

Surgery for a patient with breast cancer is postponed during the SARS outbreak, increasing the anxiety of the woman and her family about the spread of her disease.

Severe restrictions on entry to hospitals affected by SARS in the Toronto area meant that thousands of people were denied medical care, sometimes for severe illnesses such as cancer and heart disease. Some of these patients died before receiving treatment. Those who were admitted, with or without SARS, suffered loss of contact and emotional support from family and friends as hospitals closed their doors to visitors. ${ }^{5}$

Strong measures taken to contain the spread of a deadly disease will inevitably cause considerable collateral damage. Authorities in the Toronto region had to make hard choices about which medical services to maintain and which to place on hold. They had to weigh risks, benefits, and opportunity costs. How such decisions can best be made needs thorough re-evaluation. Clearly, they should be made in a fair manner, preserving as much equity as possible between the interests of patients with SARS and those who need urgent treatment for other diseases. Procedural fairness should also be ensured in making such decisions.

The collateral damage of the SARS outbreak has yet to be fully quantified and may be substantial. Health systems need to have plans for setting priorities in place for future epidemics.

\section{SARS in a globalised world}

In Guangdong province in rural China a farmer develops a severe respiratory infection. Normally, the disease would have stayed in the community, but family travels begin a chain of events that takes SARS around the world. The World Health Organization issues travel warnings for areas with large numbers of cases.

The SARS outbreak sounded a dramatic wakeup call about global interdependence and the increasing risk to global human security from the emergence and rapid spread of infectious diseases. SARS leapt the Pacific Ocean to Toronto on an aeroplane. This shows the urgent need for people around the world to adopt the value of solidarity as much for self interest as altruistic reasons. Infectious diseases can spread in either direction between poor rural areas and rich urban areas, anywhere in the world.

The SARS epidemic also showed the urgent need to strengthen the way the world deals with emerging infectious diseases. This will require new visions of global solidarity and cooperation. ${ }^{6}$

Transparency, honesty and good communications on health issues also need emphasis. It took months for China to acknowledge that it was the source of the outbreak. It is no longer acceptable for countries to hide health information that can protect others. Sharing public health information is part of maintaining the global public good of health protection, and should be encouraged and admired. 


\section{Next steps}

The lessons learnt from the ethical choices in the Toronto SARS crisis can inform efforts to control the disease elsewhere and guide our responses to future epidemics. The approach outlined here is provisional and derived from reflection on recent experiences. Nevertheless, the values identified are a useful starting point for evaluating the ethical dimensions of outbreaks of communicable disease. Our evaluation points towards a broader, public health focused approach based on a more comprehensive set of guiding values than usually considered in the ethics of interpersonal relationships.

Further research, both conceptual and empirical, is needed. As a first step we have created a decision tool derived from the values identified above (see bmj.com). Further work is required to examine how the values identified relate to moral theory. A review of professional codes and communicable disease legislation would help to clarify professional duties and define the acceptable extent of professional obligation and public health power. Additionally, public discussion about the acceptable limits to individual liberty in public health emergencies and the expected scope of healthcare workers' duty to care would help to harmonise the actions of the health community and public expectation.

We thank Sheela Basrur for comments on an earlier version of this paper.

Contributors and sources: The authors formed the working group and are scholars in bioethics who come from various disciplines, including medicine, surgery, health law, social work, teaching, nursing, and epidemiology. Several of the team members worked in directly affected hospitals and some were involved in decision making regarding the outbreak. Michael Keating drafted the report on the basis of the discussions of the working group, and it was then reviewed and revised by all authors.

\section{Summary points}

The response to the SARS epidemic by public health, governmental, and healthcare workers raised new ethical issues

Analysis of decision making in Toronto provides lessons for dealing with a global public health threat

Five key ethical issues were identified and 10 underlying values

Public discussion is required to resolve issues surrounding acceptable limits to individual liberty and healthcare workers' duty of care in an epidemic

Funding: PAS is a distinguished investigator and REGU is a new investigator of the Canadian Institutes of Health Research. Competing interests: None declared.

1 Booth CM, Matukas LM, Tomlinson GA, et al. Clinical features and shortterm outcomes of 144 patients with SARS in the greater Toronto area. JAMA (in press). http://jama.ama-assn.org/cgi/content/full/ 289.21.JOC30885v1 (accessed 26 Oct 2003).

2 Upshur RE. Principles for the justification of public health intervention. Can J Public Health 2002;93:101-3.

3 Barbera J, Macintyre A, Gostin L, Inglesby T, O'Toole T, DeAtley C, et al. Large-scale quarantine following biological terrorism in the United States: scientific examination, logistic and legal limits, and possible consequences. JAMA 2001;286:2711-7.

4 Maunder R, Hunter J, Vincent L, Bennett J, Peladeau N, Leszcz M, et al. The immediate psychological and occupational impact of the 2003 SARS outbreak in a teaching hospital. CMAJ 2003;168:1245-51.

5 Bernstein M, Hawryluck L. Challenging beliefs and ethical concepts: the collateral damage of SARS. Crit Care 2003;7:269-71.

6 Benatar SR, Daar AS, Singer PA. Global health ethics: a rationale for mutual caring. Int Affairs 2003;79:107-38.

(Accepted 15 September 2003)

\section{$Q \mathcal{E} A$}

\section{Alcohol and the genitourinary tract}

\section{Question}

Is there such a phenomenon as alcohol induced kidney pain?

Fergus J Dignan, Lyneham

\section{Answer}

Intermittent pelvi-uretereic junction obstruction (PUJO) is a reasonably common condition, and it often presents (usually in young men) as severe loin pain after drinking alcohol. The partially obstructed pelvi-ureteric junction, where the kidney drains into the upper ureter (the tube that conveys urine into the bladder), can handle the normal fluid load given to it. When the subject takes alcohol, however, the amount of urine produced suddenly and dramatically increases (a commonly observed event). The partially obstructed junction then cannot take the volume produced, and expands, causing pain-usually severe, like that caused by a kidney stone. In fact, PUJO is a differential diagnosis in stone disease.
Diagnosis requires an $\mathrm{x}$ ray examination (intravenous urography), but often a radioisotope scan is needed to show the obstruction. There are several causes of PUJO, but usually it is just a functional inability of that part of the ureter to carry the normal peristaltic wave. Treatment is surgical if the symptoms are severe and frequent enough to warrant an operation-that is, not just every time the patient goes on a bender. There's an easier solution, then.

John F Bolton clinical fellow in urology, Bristol Royal Infirmary, Bristol

http://bmj.com/cgi/qa-display/short/bmj_el;40367

This exchange was posted on the QEA A section of bmj.com. If you want to respond to the question, or ask a new question of your own, follow the link above or go to: http://bmj.com/q\&a 